\title{
Ultrafast and ultrasensitive phototransistors based on few-layered $\mathrm{HfSe}_{2}$
}

Lei Yin, Kai Xu, Yao Wen, Zhenxing Wang, Yun Huang, Feng Wang, Tofik Ahmed Shifa, Ruiqing Cheng, He Ma, and Jun $\mathrm{He}$

Citation: Appl. Phys. Lett. 109, 213105 (2016); doi: 10.1063/1.4968808

View online: http://dx.doi.org/10.1063/1.4968808

View Table of Contents: http://aip.scitation.org/toc/apl/109/21

Published by the American Institute of Physics

\section{Articles you may be interested in}

Two-dimensional semiconductor HfSe2 and MoSe2/HfSe2 van der Waals heterostructures by molecular beam epitaxy

Appl. Phys. Lett. 106, 143105143105 (2015); 10.1063/1.4917422

Air sensitivity of MoS2, MoSe2, MoTe2, HfS2, and HfSe2

Appl. Phys. Lett. 120, 125102125102 (2016); 10.1063/1.4963290

Strong electrically tunable MoTe2/graphene van der Waals heterostructures for high-performance electronic and optoelectronic devices

Appl. Phys. Lett. 109, 193111193111 (2016); 10.1063/1.4967232

Electrical characterization of multilayer $\mathrm{HfSe} 2$ field-effect transistors on $\mathrm{SiO} 2$ substrate

Appl. Phys. Lett. 106, 143108143108 (2015); 10.1063/1.4917458

Ultrahigh sensitive MoTe2 phototransistors driven by carrier tunneling

Appl. Phys. Lett. 108, 043503043503 (2016); 10.1063/1.4941001

Field-effect transistors of high-mobility few-layer SnSe2

Appl. Phys. Lett. 109, 203104203104 (2016); 10.1063/1.4967744

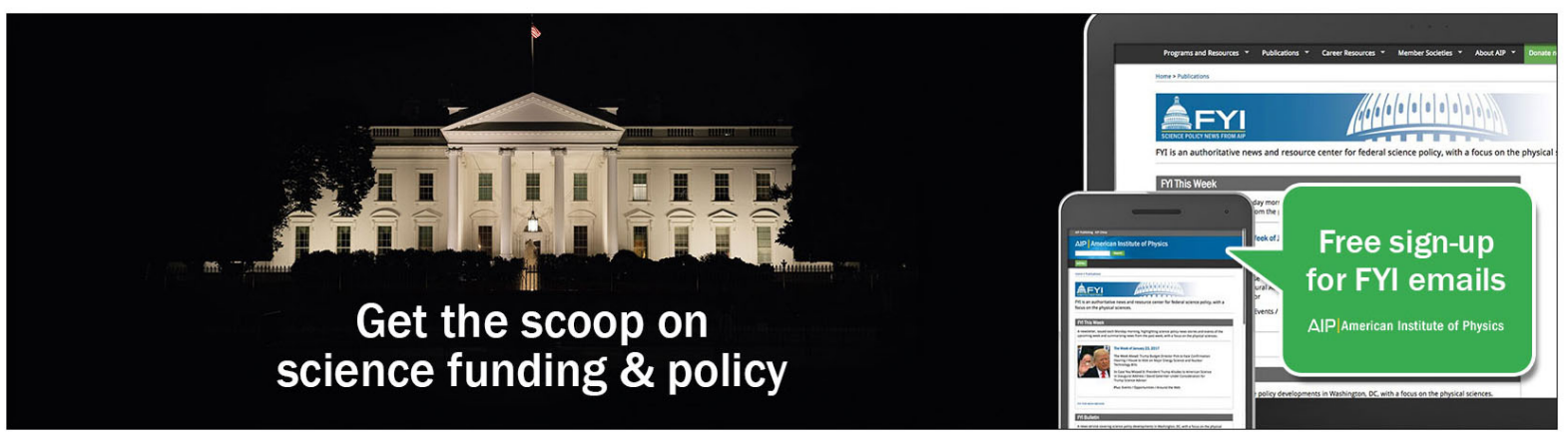




\title{
Ultrafast and ultrasensitive phototransistors based on few-layered $\mathrm{HfSe}_{2}$
}

\author{
Lei Yin, ${ }^{1,2}$ Kai Xu, ${ }^{1,2}$ Yao Wen, ${ }^{1,2}$ Zhenxing Wang, ${ }^{1}$ Yun Huang ${ }^{1,2}$ Feng Wang, ${ }^{1,2}$ \\ Tofik Ahmed Shifa, ${ }^{1,2}$ Ruiqing Cheng, ${ }^{1,2} \mathrm{He} \mathrm{Ma}^{3}$ and Jun $\mathrm{He}^{1, \mathrm{a})}$ \\ ${ }^{1}$ CAS Center for Excellence in Nanoscience, National Center for Nanoscience and Technology, \\ Beijing 100190, People's Republic of China \\ ${ }^{2}$ University of Chinese Academy of Sciences, Beijing 100049, People's Republic of China \\ ${ }^{3}$ State Key Laboratory of Low-Dimensional Quantum Physics, Department of Physics and Tsinghua-Foxconn \\ Nanotechnology Research Center, Tsinghua University, Beijing 100084, People’s Republic of China
}

(Received 5 October 2016; accepted 12 November 2016; published online 23 November 2016)

\begin{abstract}
Two-dimensional layered materials have exhibited great potential in electronic and optoelectronic applications, because of their unique chemical and physical properties. Recently, Hf-based transition metal dichalcogenides are predicted to own high room temperature mobility and are attracting increasing interest. However, only few experimental investigations are reported hitherto. In this paper, we demonstrate ultrafast and ultrasensitive back-gated $\mathrm{HfSe}_{2}$ phototransistors. Au-contacted $\mathrm{HfSe}_{2}$ phototransistors display a high on/off ratio of $10^{6}$ and modest mobilities in the range of 2.6-6.5 $\mathrm{cm}^{2} \mathrm{~V}^{-1} \mathrm{~s}^{-1}$. Additionally, the phototransistors based on $\mathrm{HfSe}_{2}$ present prominent optoelectronic performance with a high responsivity of $252 \mathrm{~A} / \mathrm{W}$ and an ultrafast response time of $7.8 \mathrm{~ms}$, implying the sensitive photoswitching behavior. Moreover, the response time can be modulated by gate voltages. The excellent field effect transistor performance coupled to the sensitive and fast photodetection makes $\mathrm{HfSe}_{2}$ have a broad application prospect for electronic and optoelectronic devices in the future. Published by AIP Publishing. [http://dx.doi.org/10.1063/1.4968808]
\end{abstract}

Two-dimensional layered materials (2DLMs) have attracted tremendous attention owing to their unique structural characteristics, physical properties, and great potential applications. ${ }^{1,2}$ As a typical material, graphene has demonstrated much novel properties in electronics, mechanics, and thermal stability. ${ }^{3,4}$ Especially, the extremely high intrinsic mobility aspired the scientific community in the area of electronic devices. ${ }^{3}$ However, the zero bandgap of intrinsic graphene would result in the high off-state current and low on/ off ratio, thereby signifying its limitation to comply with the requirements of digital logic transistors. Moreover, the weak light absorption and short photogenerated carrier lifetime of graphene limit its application in photodetection. ${ }^{5}$ In contrast, transition metal dichalcogenides (TMDs) own a sizeable bandgap that makes they are regarded as a promising candidate material for next-generation electronic and optoelectronic devices.

In the past few years, the most studied TMD material is $\mathrm{MoS}_{2} \cdot{ }^{6-8}$ For logical electronics, the monolayer $\mathrm{MoS}_{2}$ FET with a hafnium oxide gate dielectric has exhibited high carrier mobility and on/off ratio at room temperature. ${ }^{6}$ But in fact its mobility without optimization is relatively low (0.11-10 $\left.\mathrm{cm}^{2} \mathrm{~V}^{-1} \mathrm{~s}^{-1}\right)^{7,8}$ For photodetection, monolayer $\mathrm{MoS}_{2}$ possesses a direct bandgap of $1.8 \mathrm{eV}$, suggesting that it could exhibit a superior ability to detect light. A high responsivity up to $880 \mathrm{~A} / \mathrm{W}$ has been achieved in monolayer $\mathrm{MoS}_{2}$ phototransistors. ${ }^{7}$ However, the response time of $4 \mathrm{~s}$ is too slow for practical devices. Despite a faster response time of $50 \mathrm{~ms}$ previously reported, the phototransistors based on $\mathrm{MoS}_{2}$ fail to realize an impressing responsivity. ${ }^{8}$ For seeking both fast response time and high responsivity, some other 2DLMs have been studied. ${ }^{9,10}$ However, new materials and

${ }^{a)}$ Electronic mail: hej@nanoctr.cn high performance devices are still desired. Recently, Hfbased TMD materials are attracting increasing interest. ${ }^{11-13}$ Among all of the Hf-based TMDs, hafnium diselenide $\left(\mathrm{HfSe}_{2}\right)$ is predicted to be a small-bandgap semiconductor with a high electron affinity. ${ }^{14}$ Therefore, it is a promising component for forming broken-gap band alignment in vertically stacked tunnel field-effect transistors (TFETs). Additionally, theoretical calculation has demonstrated that $\mathrm{HfSe}_{2}$ owns the desirable room temperature mobility above $2500 \mathrm{~cm}^{2} \mathrm{~V}^{-1} \mathrm{~s}^{-1} \cdot{ }^{15}$ However, the experimentally reported mobility value in multilayer $\mathrm{HfSe}_{2}$ FETs is about $0.22 \mathrm{~cm}^{2}$ $\mathrm{V}^{-1} \mathrm{~s}^{-1} \cdot{ }^{13}$ Although theoretical predictions are available, adequate experimental reports are lacking that limits the tangible realization of electronic and optoelectronic properties for $\mathrm{HfSe}_{2}$-based devices.

Herein, we fabricate back-gated $\mathrm{HfSe}_{2}$ FETs using $\mathrm{Au}$ electrodes and study their electronic and optoelectronic properties. The attained mobility value at room temperature is in the range of $2.6-6.5 \mathrm{~cm}^{2} \mathrm{~V}^{-1} \mathrm{~s}^{-1}$, which is higher than that previously reported on $\mathrm{HfSe}_{2}$ and comparable to those of other back-gated 2DLMs FETs without optimization. ${ }^{8,16,17}$ Furthermore, the phototransistors based on $\mathrm{HfSe}_{2}$ present prominent optoelectronic performances in visible and nearinfrared regions. The high responsivity of $252 \mathrm{~A} / \mathrm{W}$, the gatemodulated photocurrent, and response time are achieved, indicating the ultrasensitive photoresponse property of our devices. Importantly, $\mathrm{HfSe}_{2}$ phototransistors show a quite fast response time of $0.43 \mathrm{~ms}$ that is faster than those of most other layered materials ( $5.3 \mathrm{~ms}-4 \mathrm{~s}) .^{7,8,18-20}$

The crystal structure of the $\mathrm{HfSe}_{2}$ layered compound is shown in Fig. 1(a). Hexagonal $\mathrm{HfSe}_{2}$ is composed of an atomic plane of $\mathrm{Hf}$ sandwiched between two atomic planes of Se in an octahedral configuration Se-Hf-Se. ${ }^{21}$ The atoms within each triple layer are connected through strong 
(a)
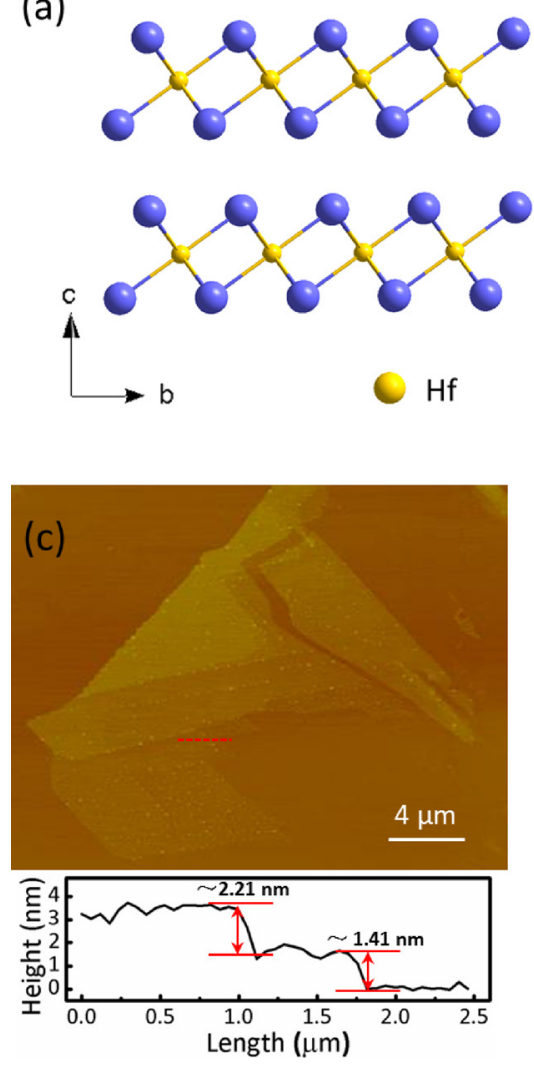
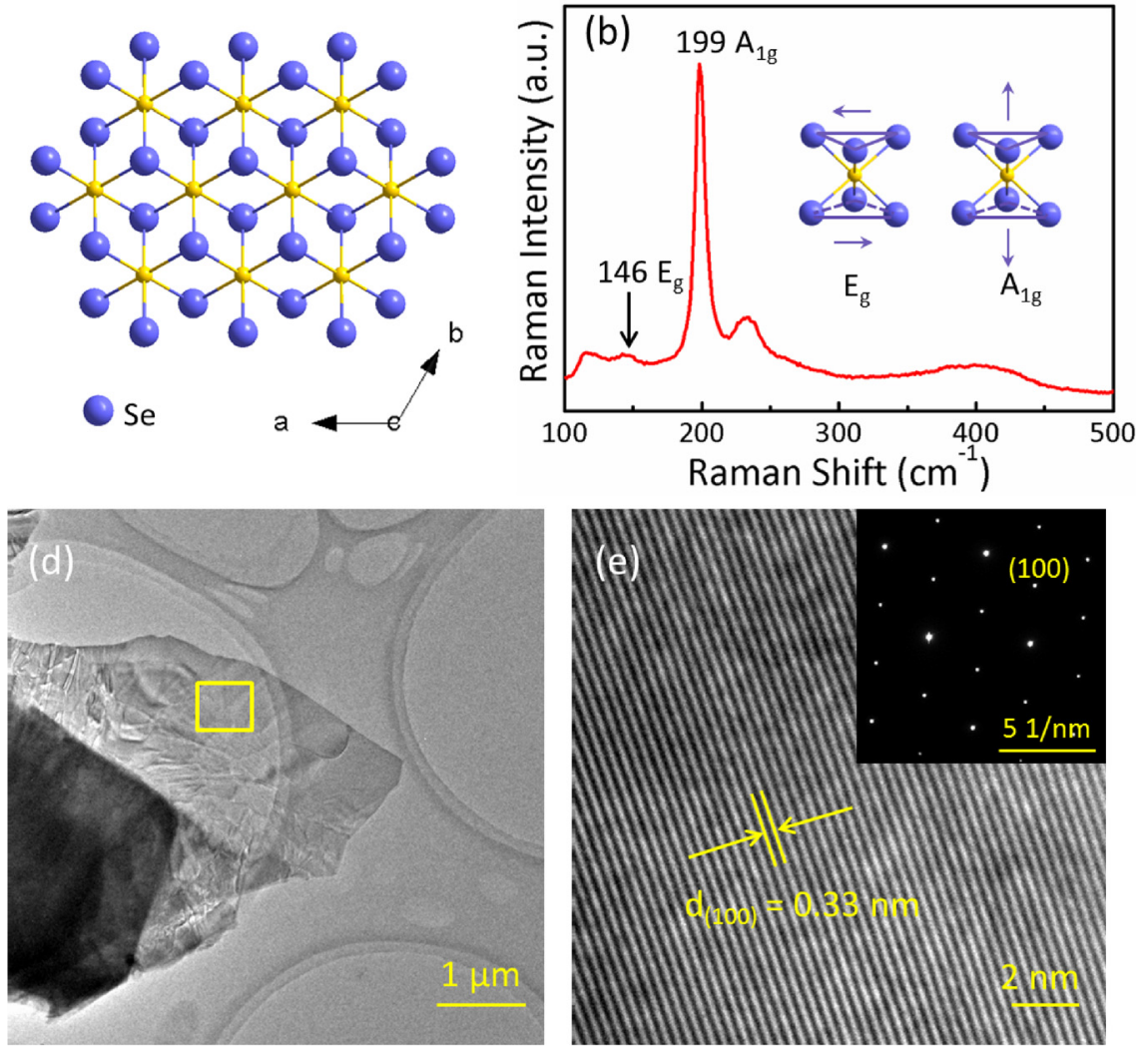

FIG. 1. (a) Side and top view of HfSe 2 crystal structures. Gold and purple spheres represent Hf and Se atoms, respectively. (b) The Raman spectra and Ramanactive modes of layered $\mathrm{HfSe}_{2}$. The purple arrows designate the vibrational directions. (c) AFM image of layered HfSe ${ }_{2}$ with different thicknesses. (d) TEM image of the $\mathrm{HfSe}_{2}$ flake at low magnification. (e) HRTEM and SAED images of the rectangular region in Fig. 1(d).

covalent bonding. However, two adjacent layers are stacked together by the weak interlayer van der Waals interaction. Figure 1(b) shows the Raman spectra of $\mathrm{HfSe}_{2}$ excited by $532 \mathrm{~nm}$ laser. Two Raman-active modes of $\mathrm{E}_{\mathrm{g}}\left(146 \mathrm{~cm}^{-1}\right)$ and $A_{1 \mathrm{~g}}\left(199 \mathrm{~cm}^{-1}\right)$ are observed, which are in agreement with previously reported results. ${ }^{12,21,22}$ The corresponding mode motions of $1 \mathrm{~T}$-phase $\mathrm{HfSe}_{2}$ are given in the inset of Fig. 1(b). We characterized the thickness and crystalline structure of $\mathrm{HfSe}_{2}$ flakes by atomic force microscopy (AFM) and transmission electron microscopy (TEM), as shown in Figures 1(c) and 1(d). The corresponding line profile in the AFM image reveals the step-like thickness of $\mathrm{HfSe}_{2}$ nanosheets from 1.41 to $3.7 \mathrm{~nm}$. The high resolution (HR)-TEM and selected-area electron diffraction (SAED) of the rectangular region in Fig. 1(d) were also performed and the results are displayed in Fig. 1(e). The interplanar spacing corresponding to the (100) plane of single-crystal $\mathrm{HfSe}_{2}$ is $0.33 \mathrm{~nm}$. Besides, the SAED pattern in the inset of Fig. 1(e) testifies the hexagonal structure of $\mathrm{HfSe}_{2}{ }^{23}$

For investigating the electronic properties of $\mathrm{HfSe}_{2}$, backgated $\mathrm{HfSe}_{2}$ FET devices were fabricated by mechanically exfoliating bulk 1T-HfSe 2 (99.995\%, HQ Graphene) onto Si substrates with $\mathrm{SiO}_{2}$ of $300 \mathrm{~nm}$. Copper grid shadow mask with gaps was covered on the surface of the Si substrate to pattern the source/drain contacts. Subsequently, $60 \mathrm{~nm} \mathrm{Au}$ was evaporated as electrodes for ohmic contacts' formation, according to the band alignment of $\mathrm{HfSe}_{2}$ (indirect bandgap of $1.1 \mathrm{eV}$ ), ${ }^{24,25}$ and work function of $\mathrm{Au}$ (supplementary material, Figure S1). The schematic representation, AFM image, and optical image (inset) of a typical $\mathrm{HfSe}_{2}$ device are illustrated in Figures 2(a) and 2(b). All measurements were performed under room temperature and vacuum condition $\left(10^{-6}\right.$ Torr). As depicted in Fig. 2(c), the HfSe 2 FET device with the thickness of $\sim 15.8 \mathrm{~nm}$ exhibits typical n-type conductance. From the measured $I_{\mathrm{ds}}-V_{\mathrm{gs}}$ curves, field effect mobility can be calculated by using the generally field-effect equation. Accordingly, the electron mobility is found to be $3 \mathrm{~cm}^{2} \mathrm{~V}^{-1} \mathrm{~s}^{-1}$. It is noted that the experimentally measured mobility is far lower than the theoretical value. This might be because of the 2-terminal measurements including the contact resistances and the existence of possible scattering mechanism. Nonetheless, the achieved mobility for our case is one order higher than that $\left(0.22 \mathrm{~cm}^{2} \mathrm{~V}^{-1} \mathrm{~s}^{-1}\right)$ previously reported on $\mathrm{HfSe}_{2}$ and comparable to those $\left(0.1-10 \mathrm{~cm}^{2} \mathrm{~V}^{-1}\right.$ $\mathrm{s}^{-1}$ ) of back-gated 2DLMs FETs without optimization. ${ }^{8,13,16,17}$ Moreover, the on/off ratio reaches $\sim 10^{6}$ that meets the required values $\left(10^{4}-10^{7}\right)$ for effective switching in digital logic transistors. ${ }^{3,26}$ Though linearly fitting the $\sqrt{I_{\mathrm{ds}}}-V_{\mathrm{gs}}$ curve of the device, we extracted its threshold voltage $\left(V_{\text {th }}\right)$ of $\sim 23.9 \mathrm{~V}$. Figure $2(\mathrm{~d})$ shows the $I_{\mathrm{ds}}-V_{\mathrm{ds}}$ curves at different gate voltages. They are linear at low drain voltages, indicating that ohmic-like contacts are formed between electrodes and $\mathrm{HfSe}_{2}$ channels. With the increase of $V_{\mathrm{ds}}$, the currents gradually become saturated, implying the onset of the "pinched-off" channel. Also, the saturation current strongly depends on gate voltage according to the CMOS square law model. In the saturation region, we can obtain a high output resistance, which is beneficial to realize 
(a)

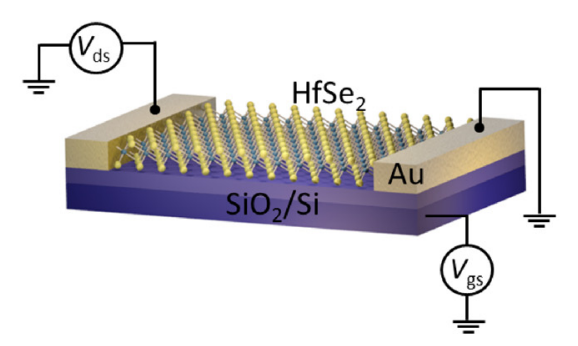

(b)

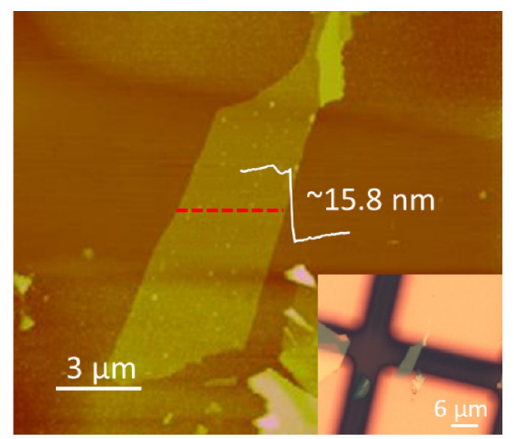

(c)

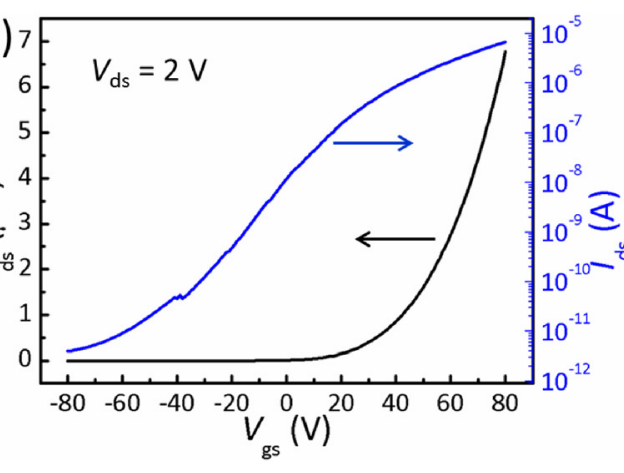

(d) 5

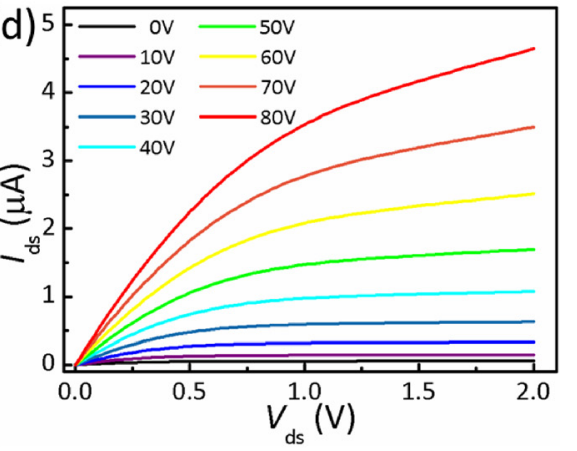

FIG. 2. (a) Device model of $\mathrm{HfSe}_{2}$ back-gated FETs. (b) AFM image and optical image (inset) of a typical $\mathrm{HfSe}_{2}$ device. The channel length and width are 6.8 and $3.6 \mu \mathrm{m}$, respectively. (c) Room-temperature transfer characteristics of the $\mathrm{HfSe}_{2}$ FET in linear and logarithmic scale. (d) Output characteristics of the corresponding device. a high voltage gain and to extract output from the input signal in digital circuits. ${ }^{27}$

Next, we study the optoelectronic characteristics of the phototransistors based on $\mathrm{HfSe}_{2}$. Figure 3(a) shows the effect of power density on $I_{\mathrm{ds}}-V_{\mathrm{gs}}$ curves under laser illumination (wavelength, $\lambda=473 \mathrm{~nm}$ ). From Fig. 3(a), photocurrent $\left(I_{\mathrm{ph}}\right)$ can be obtained by $I_{\mathrm{ph}}=I_{\text {illumination }}-I_{\text {dark }}$. We plotted the photocurrent as a function of gate voltages in Fig. 3(b). The magnitude of the $I_{\mathrm{ph}}$ increases with incident power. Furthermore, it is worth noting that the $I_{\mathrm{ph}}$ can be operated by $V_{\mathrm{gs}}$, supplying effective control for photoelectric on/off switching. Under the irradiation of laser at $248.3 \mathrm{~mW} / \mathrm{cm}^{2}$, the ratios of the illumination current to the dark current at the off state $\left(V_{\mathrm{gs}}<V_{\mathrm{th}}\right)$ are in the order of $10^{2}-10^{3}$ (supplementary material, Figure S2(a)). As illustrated in Fig. 3(c), we calculated the responsivity $\left(R=I_{\mathrm{ph}} / P S\right.$, where $P$ is the incident power density and $S$ is the effective illumination area). The highest responsivity of $252 \mathrm{~A} / \mathrm{W}$ is obtained at $P=2.6 \mathrm{~mW} / \mathrm{cm}^{2}$ and $V_{\mathrm{gs}}=80 \mathrm{~V}$. It is worth noting that the responsivity shows opposite trends with increasing laser power for positive and negative gate voltages. With the increase of laser power, $R$ decreased at $V_{\mathrm{gs}}>0 \mathrm{~V}$. In contrast, it slightly increased with power densities at $V_{\mathrm{gs}}<0 \mathrm{~V}$. Through power law fitting, it can be seen that the exponent gradually changes from negative of -0.7 at $V_{\mathrm{gs}}=80 \mathrm{~V}$ to positive of 0.2 at $V_{\mathrm{gs}}=-80 \mathrm{~V}$. This transition further reflects
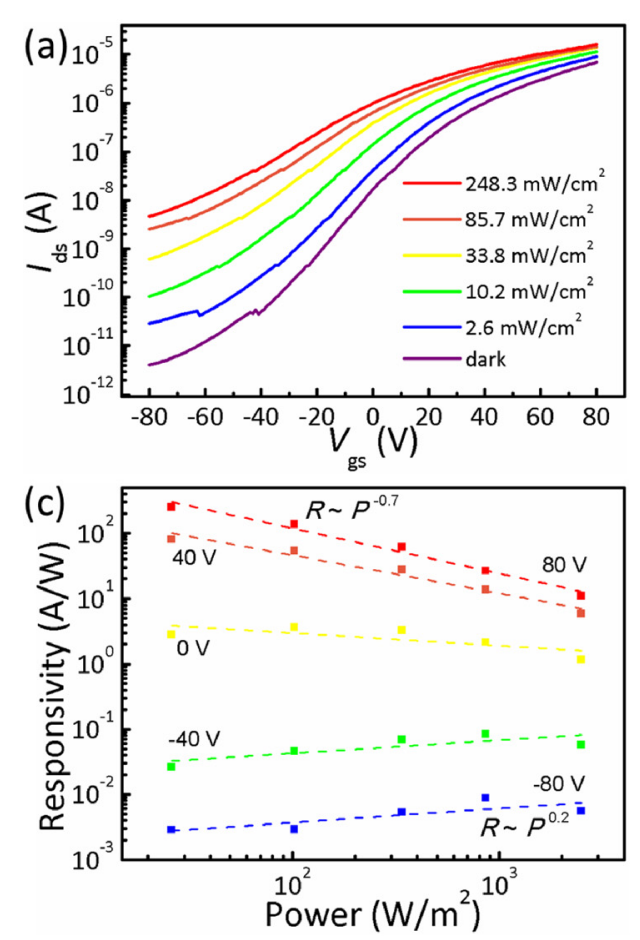
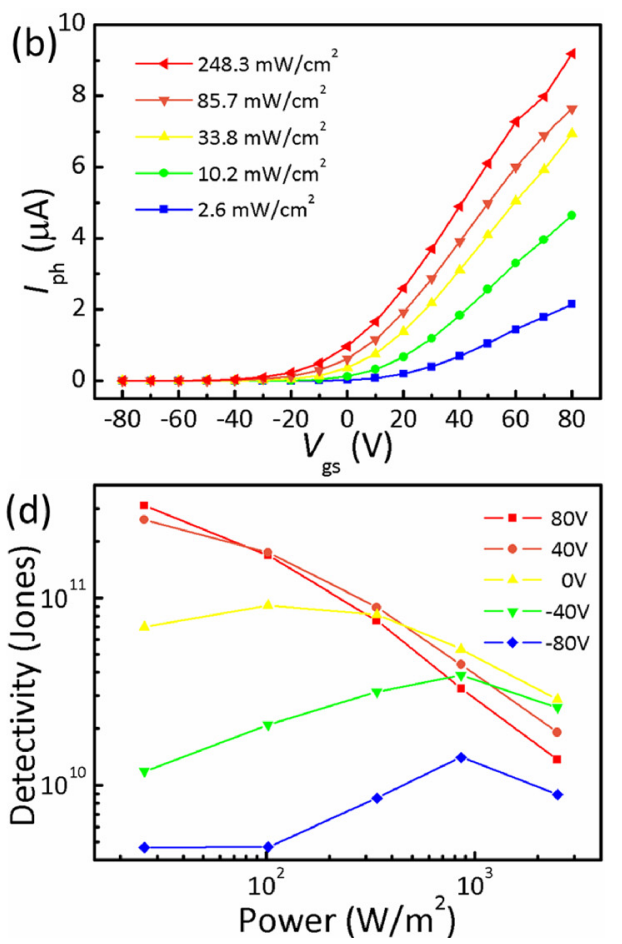

FIG. 3. (a) and (b) Transfer characteristic curves and photocurrent of $\mathrm{HfSe}_{2}-$ based phototransistor under various illumination power densities of laser. The responsivity (c) and detectivity (d) of $\mathrm{HfSe}_{2}$ phototransistor change with the laser power at different gate voltages. 
the opposite trends for positive and negative gate voltages. Generally, photoresponse is closely relevant to trap states and lifetime of photogenerated carriers. ${ }^{7,28}$ Here, the opposite trends on responsivity with light power may originate from different scattering intensity and recombination rates of photogenerated carriers. At $V_{\mathrm{gs}}>0 \mathrm{~V}$, the device is in the on state and possesses high carrier density. When increasing the light power, scattering and recombination of photogenerated carriers are largely enhanced, and thus the responsivity decreases. On the contrary, the device is in the off state and owns low carrier density at $V_{\mathrm{gs}}<0 \mathrm{~V}$. The scattering intensity and recombination rate of photogenerated carriers have no obvious changes with power density increasing; thus, the responsivity exhibits a slight increase. Besides, specific detectivity $\left(D^{*}\right)$ is also estimated by using $D^{*}=R S^{1 / 2} /$ $\left(2 q I_{\mathrm{d}}\right)^{1 / 2}$, ${ }^{29}$ where $q$ and $I_{\mathrm{d}}$ are the electron charge and the dark current respectively. Figure 3(d) displays the calculated $D^{*}$ values. The maximal $D^{*}$ over $10^{11}$ Jones is acquired at $P=2.6 \mathrm{~mW} / \mathrm{cm}^{2}, V_{\mathrm{gs}}=80 \mathrm{~V}$. These results manifest that our $\mathrm{HfSe}_{2}$ phototransistors are comparable to others based on 2DLMs. ${ }^{818-20,27}$ We also calculated photogain $(G)$, another important parameter, which is depicted in Fig. S2(b) of the supplementary material.

With the purpose of studying the photoswitching behavior, we alternately turned on and off a laser with a density of $248.3 \mathrm{~mW} / \mathrm{cm}^{2}$. The time-resolved photoresponse is shown in Fig. 4(a). The stable current for multiple illumination cycles at $V_{\mathrm{gs}}=0 \mathrm{~V}$ reflects the reliability of our $\mathrm{HfSe}_{2}$-based phototransistors. Figure 4 (b) illustrates the single photocurrent on/off switching cycle at $V_{\mathrm{gs}}=-80 \mathrm{~V},-40 \mathrm{~V}, 0 \mathrm{~V}$, and $40 \mathrm{~V}$. It can be noted that the currents rise slowly after turning on the laser at $V_{\mathrm{gs}}=-80 \mathrm{~V}$ (off state). However, the photocurrents at $V_{\mathrm{gs}}=40 \mathrm{~V}$ (on state) rise fast to reach steady saturation under laser illumination. This phenomenon will be discussed later. To extract rise time, we define it as the required time that photocurrent reaches $90 \%$ of the entire current increment. Interestingly, it is found that rise time exhibits the gate-modulated behavior. As shown in Fig. 4(c), it decreases with the increase of gate voltages. Response time modulation is generally associated with various factors. ${ }^{11,28,30}$ Here, we attribute the result to the Schottky barrier and depletion region between channel materials and electrodes.

As is well known, when a fixed voltage is exerted on the drain terminal of Schottky transistors, voltage drop primarily acts on the reverse Schottky barrier. ${ }^{31-34}$ If a Schottky barrier reduces, it will cause the shrinkage of the corresponding depletion region. Conversely, the depletion region can be broadened with the increase of Schottky barrier. Besides, it has been demonstrated that the band tail between channel and metal contacts decays exceptionally slowly, and the depletion region can broaden to several micrometers in the channel materials. ${ }^{31,35}$ According to the band alignment of $\mathrm{HfSe}_{2}$ and work function of $\mathrm{Au}$, the bands near source and drain electrodes bend downward at the off state (Fig. 4(d)-I). When a bias voltage $\left(V_{\mathrm{ds}}=2 \mathrm{~V}\right)$ was applied, the reverse Schottky barrier (near source terminal) would be lowered. This would cause the depletion region near the source electrode $\left(\mathrm{W}_{\mathrm{S}}\right)$ to be dramatically shrunken so that it can be ignorable. Another depletion region near the drain electrode $\left(\mathrm{W}_{\mathrm{D}}\right)$ would not significantly change, as displayed in Fig. 4(d)-II. Therefore, there is a short depletion region with weak built-in electric field, generating a long rise time. It is attributed to the inefficient separation of excited electronhole pairs. Moreover, it would also lead to voltage drop related to defects, and charge impurities in the channel become striking. Hence, a current rising stage is observed after turning on the laser at $V_{\mathrm{gs}}<V_{\text {th }}$ (Fig. 4(b)), resulting from the enhancement photocurrent component associated with photodesorption or potoexcitation via defects or charge impurity states to band edge. In contrast, the bands at both ends of the channel slightly bend upward at the on state (Fig. 4(d)-III). Under the impact of enough drain-source voltage, the $\mathrm{W}_{\mathrm{S}}$ would be broadened. Meanwhile, such a slight bend at the drain electrode is easy to be reversed by the applied $V_{\mathrm{ds}}$, causing the emergence of a wide depletion region. Thus, there are two depletion regions in the channel (Fig. 4(d)-IV), both of which generating the fast rise time. Despite the
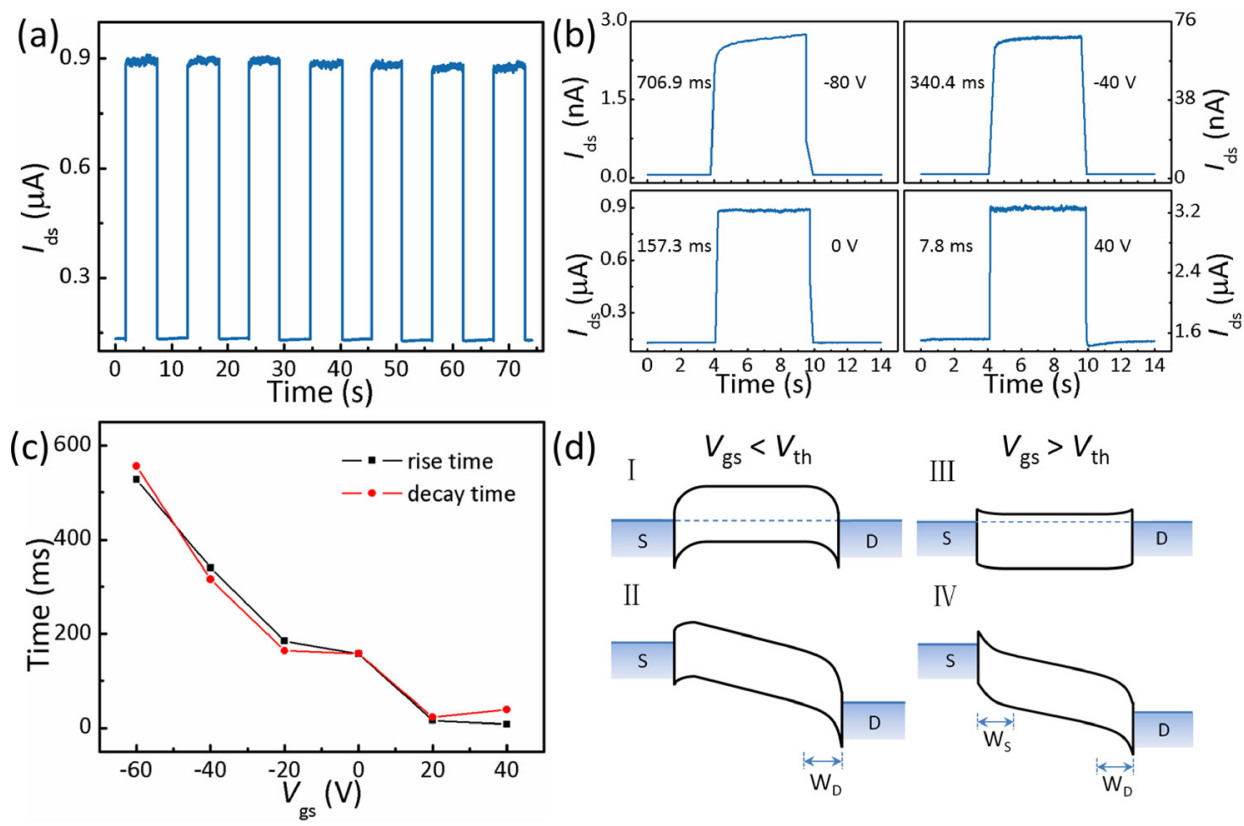

(d)

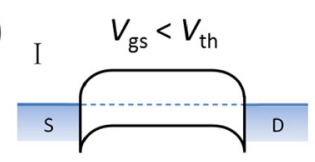

II

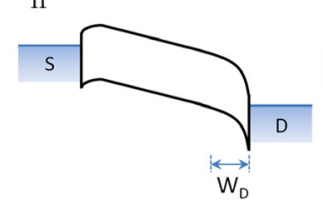

$V_{\mathrm{gs}}>V_{\mathrm{th}}$
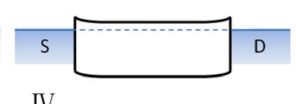

IV

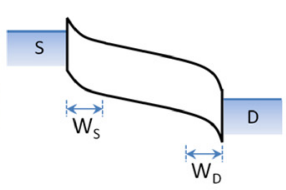

FIG. 4. (a) The time-resolved photoresponse of the $\mathrm{HfSe}_{2}$ phototransistor. (b) The single photocurrent on/off switching cycle at various gate voltages. (c) The extracted rise time (black) and decay time (red) reveal the gate-modulated trend. (d) Band diagram at the off state $\left(V_{\mathrm{gs}}<V_{\mathrm{th}}\right)$ and the on state $\left(V_{\mathrm{gs}}>V_{\mathrm{th}}\right)$. 
limitation in our measurement setup, the rise time as fast as $7.8 \mathrm{~ms}$ is obtained. It is considerable that the value is comparable, even faster than those $(5.3 \mathrm{~ms}-4 \mathrm{~s})$ of most phototransistors based on 2DLMs. ${ }^{7,8,18-20}$ We also measured another thicker device using a $20 \mathrm{~ms}$ pulse train of voltage and an optical chopper. The ultrafast rise time of $0.43 \mathrm{~ms}$ is realized, as shown in Figure S3 (supplementary material). The decay time is also extracted from maximum to $10 \%$ of the peak output value, as illustrated in Fig. 4(c). We noted that decay time shows decreasing trend with the increase of gate voltages. As mentioned above, the photocurrent may be mainly associated with defects and charge impurity states at $V_{\mathrm{gs}}<V_{\mathrm{th}}$, causing the slower decay time. We believe that such excellent photoresponse (high responsivity and fast response time), superior than many other TMD phototransistors, originates from especially easy acquisition and extraction of photogenerated carriers due to the high absorption coefficient and high mobility of $\mathrm{HfSe}_{2} \cdot{ }^{15,36}$ Additionally, the response of the $\mathrm{HfSe}_{2}$-based phototransistor with a nearinfrared laser $(\lambda=800 \mathrm{~nm})$ and ultraviolet-visible diffuse reflectance spectra of $\mathrm{HfSe}_{2}$ are demonstrated in Figs. S4 and S5 of the supplementary material.

In conclusion, the phototransistors based on $\mathrm{HfSe}_{2}$ are fabricated. Subsequently, the electronic and optoelectronic properties of them are investigated. The n-type $\mathrm{HfSe}_{2} \mathrm{FETs}_{\mathrm{s}}$ exhibit high on/off ratios of $10^{6}$ and modest mobilities in the range of $2.6-6.5 \mathrm{~cm}^{2} \mathrm{~V}^{-1} \mathrm{~s}^{-1}$. Moreover, the $\mathrm{HfSe}_{2}$-based phototransistors reveal desirable photoresponse in visible and near-infrared regions. The maximal responsivity at $V_{\mathrm{gs}}=80 \mathrm{~V}$ is $252 \mathrm{~A} / \mathrm{W}$ for a laser power of $2.6 \mathrm{~mW} / \mathrm{cm}^{2}$ $(\lambda=473 \mathrm{~nm})$ and $3961 \mathrm{~A} / \mathrm{W}$ for a laser power of $0.12 \mathrm{~mW} /$ $\mathrm{cm}^{2}(\lambda=800 \mathrm{~nm})$. The sensitive photoswitching behavior of the phototransistors can be corroborated from the observed ultrafast rise time of $0.43 \mathrm{~ms}$. Owing to these superb performance, $\mathrm{HfSe}_{2}$ will have a broad application prospect for electronic and optoelectronic devices in the future.

See supplementary material for detailed band alignment and more photoresponse data.

This work was supported by the National Natural Science Foundation of China (Nos. 21373065, 61474033, and 61574050), Strategic Priority Research Program of the Chinese Academy of Sciences (Grant No. XDA09040201), 973 Program of the Ministry of Science and Technology of China (No. 2012CB934103), and CAS Key Laboratory of Nanosystem and Hierarchical Fabrication. The authors also gratefully acknowledge the support of Youth Innovation Promotion Association CAS.

${ }^{1}$ F. H. Koppens, T. Mueller, P. Avouris, A. C. Ferrari, M. S. Vitiello, and M. Polini, Nat. Nanotechnol. 9, 780 (2014).

${ }^{2}$ Y. Zhang, B. Zheng, C. Zhu, X. Zhang, C. Tan, H. Li, B. Chen, J. Yang, J. Chen, Y. Huang, L. Wang, and H. Zhang, Adv. Mater. 27, 935 (2015).

${ }^{3}$ F. Schwierz, Nat. Nanotechnol. 5, 487 (2010).
${ }^{4}$ C. Lee, X. Wei, J. W. Kysar, and J. Hone, Science 321, 385 (2008).

${ }^{5}$ M. Breusing, C. Ropers, and T. Elsaesser, Phys. Rev. Lett. 102, 086809 (2009).

${ }^{6}$ B. Radisavljevic, A. Radenovic, J. Brivio, V. Giacometti, and A. Kis, Nat. Nanotechnol. 6, 147 (2011).

${ }^{7}$ O. Lopez-Sanchez, D. Lembke, M. Kayci, A. Radenovic, and A. Kis, Nat. Nanotechnol. 8, 497 (2013).

${ }^{8}$ Z. Yin, H. Li, H. Li, L. Jiang, Y. Shi, Y. Sun, G. Lu, Q. Zhang, X. Chen, and H. Zhang, ACS Nano 6, 74 (2012).

${ }^{9}$ M. Huang, M. Wang, C. Chen, Z. Ma, X. Li, J. Han, and Y. Wu, Adv. Mater. 28, 3481 (2016).

${ }^{10}$ J. O. Island, S. I. Blanter, M. Buscema, H. S. J. van der Zant, and A. Castellanos-Gomez, Nano Lett. 15, 7853 (2015).

${ }^{11}$ K. Xu, Z. Wang, F. Wang, Y. Huang, F. Wang, L. Yin, C. Jiang, and J. He, Adv. Mater. 27, 7881 (2015).

${ }^{12}$ R. Yue, A. T. Barton, H. Zhu, A. Azcatl, L. F. Pena, J. Wang, X. Peng, N. Lu, L. Cheng, R. Addou, S. McDonnell, L. Colombo, J. W. Hsu, J. Kim, M. J. Kim, R. M. Wallace, and C. L. Hinkle, ACS Nano 9, 474 (2015).

${ }^{13}$ M. Kang, S. Rathi, I. Lee, D. Lim, J. Wang, L. Li, M. A. Khan, and G.-H. Kim, Appl. Phys. Lett. 106, 143108 (2015).

${ }^{14}$ C. Gong, H. Zhang, W. Wang, L. Colombo, R. M. Wallace, and K. Cho, Appl. Phys. Lett. 103, 053513 (2013).

${ }^{15}$ W. Zhang, Z. Huang, W. Zhang, and Y. Li, Nano Res. 7, 1731 (2014).

${ }^{16}$ S. Larentis, B. Fallahazad, and E. Tutuc, Appl. Phys. Lett. 101, 223104 (2012).

${ }^{17}$ D. J. Late, B. Liu, J. Luo, A. Yan, H. S. Matte, M. Grayson, C. N. Rao, and V. P. Dravid, Adv. Mater. 24, 3549 (2012).

${ }^{18}$ S. R. Tamalampudi, Y. Y. Lu, U. R. Kumar, R. Sankar, C. D. Liao, B. K. Moorthy, C. H. Cheng, F. C. Chou, and Y. T. Chen, Nano Lett. 14, 2800 (2014).

${ }^{19}$ P. Hu, L. Wang, M. Yoon, J. Zhang, W. Feng, X. Wang, Z. Wen, J. C. Idrobo, Y. Miyamoto, D. B. Geohegan, and K. Xiao, Nano Lett. 13, 1649 (2013).

${ }^{20}$ N. Perea-López, A. L. Elías, A. Berkdemir, A. Castro-Beltran, H. R. Gutiérrez, S. Feng, R. Lv, T. Hayashi, F. López-Urías, S. Ghosh, B. Muchharla, S. Talapatra, H. Terrones, and M. Terrones, Adv. Funct. Mater. 23, 5511 (2013).

${ }^{21}$ G. Lucovsky, R. M. White, J. A. Benda, and J. F. Revelli, Phys. Rev. B 7, 3859 (1973).

${ }^{22}$ A. Cingolani, M. Lugara, and F. Levy, Phys. Scr. 37, 389 (1988).

${ }^{23}$ D. T. Hodul and A. M. Stacy, J. Solid State Chem. 54, 438 (1984).

${ }^{24}$ D. Tsoutsou, K. E. Aretouli, P. Tsipas, J. Marquez-Velasco, E. Xenogiannopoulou, N. Kelaidis, S. Aminalragia Giamini, and A. Dimoulas, ACS Appl. Mater. Interfaces 8, 1836 (2016).

${ }^{25}$ K. E. Aretouli, P. Tsipas, D. Tsoutsou, J. Marquez-Velasco, E. Xenogiannopoulou, S. A. Giamini, E. Vassalou, N. Kelaidis, and A. Dimoulas, Appl. Phys. Lett. 106, 143105 (2015).

${ }^{26}$ P. Avouris, Z. Chen, and V. Perebeinos, Nat. Nanotechnol. 2, 605 (2007).

${ }^{27}$ W. Choi, M. Y. Cho, A. Konar, J. H. Lee, G. B. Cha, S. C. Hong, S. Kim, J. Kim, D. Jena, J. Joo, and S. Kim, Adv. Mater. 24, 5832 (2012).

${ }^{28}$ M. M. Furchi, D. K. Polyushkin, A. Pospischil, and T. Mueller, Nano Lett. 14, 6165 (2014).

${ }^{29}$ W. Zhang, M. H. Chiu, C. H. Chen, W. Chen, L. J. Li, and A. T. Wee, ACS Nano 8, 8653 (2014).

${ }^{30}$ M. Buscema, J. O. Island, D. J. Groenendijk, S. I. Blanter, G. A. Steele, H. S. van der Zant, and A. Castellanos-Gomez, Chem. Soc. Rev. 44, 3691 (2015).

${ }^{31}$ M. Freitag, J. C. Tsang, A. Bol, D. Yuan, J. Liu, and P. Avouris, Nano Lett. 7, 2037 (2007).

${ }^{32}$ Z. Y. Zhang, C. H. Jin, X. L. Liang, Q. Chen, and L. M. Peng, Appl. Phys. Lett. 88, 073102 (2006).

${ }^{33}$ Z. Fan and J. G. Lu, Appl. Phys. Lett. 86, 032111 (2005).

${ }^{34}$ A. Bachtold, M. S. Fuhrer, S. Plyasunov, M. Forero, E. H. Anderson, A. Zettl, and P. L. McEuen, Phys. Rev. Lett. 84, 6082 (2000).

${ }^{35}$ F. Leonard and J. Tersoff, Phys. Rev. Lett. 83, 5174 (1999).

${ }^{36}$ C. Gaiser, T. Zandt, A. Krapf, R. Serverin, C. Janowitz, and R. Manzke, Phys. Rev. B 69, 075205 (2004). 